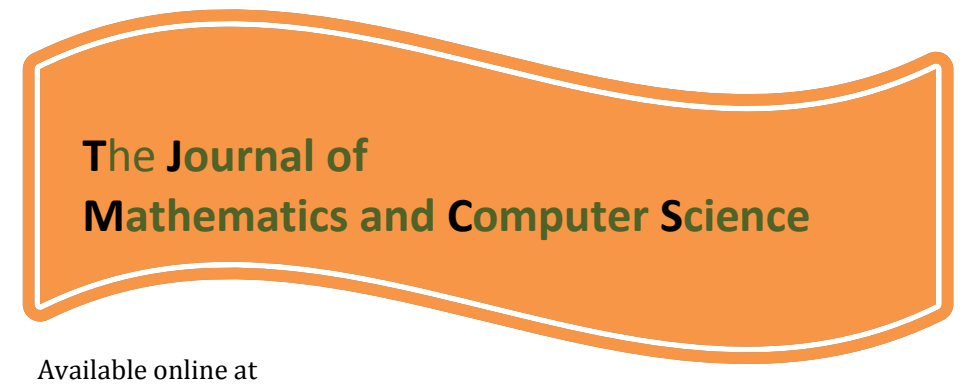

\title{
http://www.TJMCS.com
}

The Journal of Mathematics and Computer Science Vol. 4 No.3 (2012) 402 - 410

\section{T-age Replacement Policy in Fuzzy Renewal Reward Processes}

\author{
Behrouz Fathi Vajargah ${ }^{1, *}$, Sara Ghasemalipour ${ }^{2}$ \\ ${ }^{1}$ Department of Statistics, Faculty of Mathematical Sciences, University of Guilan, \\ Iran,.fathi@guilan.ac.ir, \\ ${ }^{2}$ Department of Statistics, Faculty of Mathematical Sciences, University of Guilan, Iran, \\ s.ghasemalipour@gmail.com
}

Received: February 2012, Revised: May 2012

Online Publication: July 2012

\begin{abstract}
This paper studies a renewal reward process with fuzzy reward and fuzzy random inter arrival times. A theorem about the long run average fuzzy reward and fuzzy life time is proved. The original problem is evaluating the membership of the long run average fuzzy cost per unit time that for obtaining membership, we should solve a nonlinear programming problem. Finally, some application example is provided to illustrate the result.
\end{abstract}

Keywords: Fuzzy renewal reward processes, Fuzzy random reward, Fuzzy random variables, Membership function, Fuzzy life time, Nonlinear programming

\section{Introduction}

Renewal reward processes are an important type of renewal models. The stochastic renewal reward theorem is one of the most important results in this area. On the other hand, several researches recently studied on fuzzy renewal processes. Zhao and Liu [1] discussed a fuzzy renewal process generated by a sequence of i.i.d positive fuzzy variables obtained a fuzzy elementary renewal theorem and a fuzzy renewal reward theorem. Wang and Watada [2] discuss a renewal reward process with fuzzy random interarrival times and rewards under the independence with t-norms (T-independence), which induces the (generalized) t-norm-based extension principle for the operations of fuzzy realizations of fuzzy random variables. There are many approaches taken in the literature to decide on a maintenance strategy. For a system which consists of one component see Valdez-Florez [3] for an extensive review. Different authors assume different cost structures. Usually the costs are either constants or random variables. Constant costs will not contribute anything to modeling imprecision in the

\footnotetext{
$1,{ }^{*}$ Corresponding author: Position and Special field of the first author

${ }^{2}$ Position and Special field of the second author
} 


\section{Behrouz Fathi Vajargah, Sara Ghasemalipour/ TJMCS Vol. 4 No. 3 (2012) 402 - 410}

market place; random costs assumption is valid under the condition that market behavior can be fully characterized in the context of probability measures.

In this paper, first in section2, we describe some basic definitions of fuzzy theory like fuzzy numbers, fuzzy random variables ... .In section 3, we discuss the renewal reward process when the reward and inter arrival time cannot be known exactly. We regard to the prices as fuzzy numbers and the life time of a switch as a fuzzy random variable. Then we prove a theorem about the long-run average fuzzy reward and fuzzy inter arrival time. In section 4 , we introduce a $T$-age replacement policy and we propose to find $T$ age such that coast to be minimum. Finally in section 5 an example is considered.

\section{Fuzzy numbers and fuzzy variables}

Some basic definitions of fuzzy numbers and fuzzy random variables are stated here.

If $\Omega$ is some set, then a fuzzy subset $\bar{A}$ of $\Omega$ is defined by its membership function, written by $\mu_{\bar{A}}(x)$, which produces values in [0,1] for all $x$ in $\Omega$. So, $\mu_{\bar{A}}(x)$ is a function mapping $\Omega$ into [0,1]. When $\mu_{\bar{A}}(x)$ is always equal to one or zero we obtain a crisp (nonfuzzy) subset of $\Omega$.

A general definition of fuzzy number may be found in ([4],[5]), however our fuzzy numbers will be almost, fuzzy numbers. A triangular fuzzy number $N$ is defined by three numbers $a<b<c$ where the base of the triangle is the interval $[a, c]$ and its vertex is at $x=b$. Triangular fuzzy numbers will be written as $N=(a / b / c)$.

Alpha-cuts are slices through a fuzzy set producing regular (non-fuzzy) sets. If $\bar{A}$ is a fuzzy subset of some set $\Omega$, then an $\alpha$-cut of $\bar{A}$, written $\bar{A}[\alpha]$ is defined as

$$
\bar{A}[\alpha]=\left\{x \in \Omega \mid \mu_{\bar{A}}(x) \geq \alpha\right\},
$$

for all $\alpha, 0<\alpha \leq 1$.

$\bar{A}$ is called convex fuzzy set if $\mu_{\bar{A}}[\lambda x+(1-\lambda) y] \geq \min \{\bar{A}(x), \bar{A}(y)\}$ for $\lambda \in[0,1]$.

Let $\bar{A}$ be a fuzzy set with membership function $\mu_{\bar{A}}(x)$ and $\bar{A}[\alpha]=\left\{x \in \Omega \mid \mu_{\bar{A}}(x) \geq \alpha\right\}$, Then

$$
\mu_{\bar{A}}(x)=\sup _{\alpha \in[0,1]} \alpha 1_{A_{\alpha}}(x),
$$

where

$$
1_{A_{\alpha}}(x)=\left\{\begin{array}{cc}
1, & x \in A_{\alpha} \\
o, & \text { o.w. }
\end{array}\right.
$$

Fuzzy variable: Let $\Theta$ be a nonempty set, and $P(\Theta)$ shows the power set of $\Theta$. In order to present the axiomatic definition of possibility, Nahmias [6] and Liu [7] gave the following four axioms:

Axiom 1. $\operatorname{Pos}\{\Theta\}=1$.

Axiom 2. $\operatorname{Pos}\{\phi\}=0$.

Axiom3. $\operatorname{Pos}\left\{\bigcup_{i} A_{i}\right\}=\sup _{i} \operatorname{Pos}\left\{A_{i}\right\}$ for any collection $A_{i}$ in $P(\Theta)$.

Axiom 4. Let $\Theta_{i}$ be nonempty sets on which $\operatorname{Pos}_{i}\{$. $\}$ satisfies the first three axioms, $i=1,2, \ldots, n$, respectively, and $\Theta=\Theta_{1} \times \Theta_{2} \times \ldots \times \Theta_{n}$. Then

$$
\operatorname{Pos}\{A\}=\sup _{\left(\theta_{1}, \ldots \theta_{n}\right) \in A} \operatorname{Pos}_{1}\left\{\theta_{1}\right\} \wedge \operatorname{Pos}_{2}\left\{\theta_{2}\right\} \wedge \ldots \wedge \operatorname{Pos}_{n}\left\{\theta_{n}\right\}
$$




\section{Behrouz Fathi Vajargah, Sara Ghasemalipour/ TJMCS Vol. 4 No. 3 (2012) 402 - 410}

for each $A \in P(\Theta)$.

Definition 1. (Liu and Liu [8]). Let $\Theta$ be a nonempty set, and $P(\Theta)$ the power set of $\Theta$. Then the set function Pos is called a possibility measure, if it satisfies the first three axioms, and $(\Theta, P(\Theta), P o s)$ is called a possibility space.

Definition 2. Let $(\Theta, P(\Theta), P o s)$ be a possibility space, and $A$ presents a set in $P(\Theta)$. Then the necessity measure of $A$ is defined by:

$$
\operatorname{Nec}\{A\}=1-\operatorname{Pos}\left\{A^{c}\right\} .
$$

Definition 3. (Liu and Liu [8]). Let $(\Theta, P(\Theta), P o s)$ be a possibility space, and $A$ be a set in $P(\Theta)$. Then the credibility measure of $A$ is defined by:

$$
\operatorname{Cr}\{A\}=\frac{1}{2}(\operatorname{Pos}\{A\}+N e c\{A\}) .
$$

Definition 4. A fuzzy variable $\xi$ is defined as a function from the possibility space $(\Theta, P(\Theta), P o s)$ to the set of real numbers, and its membership function is derived by:

$$
\mu_{\xi}(r)=\operatorname{Pos}\{\theta \in \Theta \mid \xi(\theta)=r\} .
$$

Definition 5. (Liu and Liu [8]). Let $\xi$ be a fuzzy variable on the possibility space $(\Theta, P(\Theta), P o s)$. Then the expected value $E[\xi]$ is defined by:

$$
E[\xi]=\int_{0}^{\infty} C r\{\xi \geq r\} d r-\int_{-\infty}^{0} C r\{\xi \leq r\} d r,
$$

provided that at least one of the two integrals is finite. In particular, if the fuzzy variable $\xi$ is positive (i.e. $\operatorname{Pos}\{\xi \leq 0\}=0)$, then

$$
E[\xi]=\int_{-\infty}^{0} C r\{\xi \leq r\} d r
$$

\section{Fuzzy renewal reward processes}

Consider a renewal process $N(t), t>0$ having inter arrival times $\tilde{X}_{n}$ for $n \geq 1$, and $\underset{\sim}{\text { suppose that }} \tilde{X}_{n}$ is a random fuzzy variable and each time a renewal occurs we receive a reward. We denote by $\widetilde{R}_{n}$, the fuzzy reward earned at the time of the $n$-th renewal, where $\tilde{R}_{n}$ is a fuzzy random variable. We shall assume that the $\tilde{R}_{n}$ for $n \geq 1$ are independent and identically distributed. If we let

$$
\tilde{R}(t)=\oplus_{n=1}^{N(t)} \tilde{R}_{n},
$$

then $\tilde{R}(t)$ represents the total fuzzy reward earned by time $t$. Then, $\left(\tilde{R}_{n}\right)_{\alpha}^{L}$ and $\left(\tilde{R}_{n}\right)_{\alpha}^{U}$ are random variables for all $\alpha[9]$. Thus we have

$$
\begin{gathered}
E\left[\tilde{R}_{\alpha}^{L}\right]=E\left[\left(\tilde{R}_{n}\right)_{\alpha}^{L}\right], \\
E\left[\tilde{R}_{\alpha}^{U}\right]=E\left[\left(\tilde{R}_{n}\right)_{\alpha}^{U}\right], \\
E[\tilde{X}]=E\left[\tilde{X}_{n}\right] .
\end{gathered}
$$

Theorem 3.1 Let $A_{\alpha}^{L}=E\left[\tilde{R}_{\alpha}^{L}\right] / E[\tilde{X}]$ and $A_{\alpha}^{U}=E\left[\tilde{R}_{\alpha}^{U}\right] / E[\tilde{X}]$. Let $\bar{A}$ be a fuzzy number with membership function 


$$
\xi_{\bar{A}}(r)=\sup _{0 \leq \alpha \leq 1} \alpha I_{\left[A_{\alpha}^{L}, A_{\alpha}^{U}\right]}(r) .
$$

Suppose that $E\left[\tilde{R}_{\alpha}^{L}\right]<\infty, E\left[\tilde{R}_{\alpha}^{U}\right]<\infty$ and $E[\tilde{X}]<\infty$ for all $\alpha$. Then $\tilde{R}(t) / t \rightarrow \tilde{A}$ with probability one level-wise . If $A_{\alpha}^{L}$ and $A_{\alpha}^{L}$ are left continuous with respect to $\alpha$.

Proof. We can rewrite $\tilde{R}(t) / t$ to following form

$$
(\tilde{R}(t) / t)_{\alpha}^{L}=\frac{\sum_{n=1}^{N(t)}\left(\tilde{R}_{n}\right)_{\alpha}^{L}}{t}=\left[\frac{\sum_{n=1}^{N(t)}\left(\tilde{R}_{n}\right)_{\alpha}^{L}}{N(t)}\right]\left[\frac{N(t)}{t}\right] .
$$

By the strong law of large numbers, we obtain that

$$
\left[\frac{\sum_{n=1}^{N(t)}\left(\tilde{R}_{n}\right)_{\alpha}^{L}}{N(t)}\right] \rightarrow E\left[\tilde{R}_{\alpha}^{L}\right]
$$

with probability one as $t \rightarrow \infty$.

Similarly, we have

with probability one as $t \rightarrow \infty$.

$$
\left[\frac{\sum_{n=1}^{N(t)}\left(\tilde{R}_{n}\right)_{\alpha}^{U}}{N(t)}\right] \rightarrow E\left[\tilde{R}_{\alpha}^{U}\right]
$$

Applying the fact that

$$
\frac{N(t)}{t} \rightarrow \frac{1}{E[\tilde{X}]}
$$

([10]) with probability one as $t \rightarrow \infty$ we get the desired result.

We say that a cycle is complete every time if a renewal occurs, then Theorem 3.1 presents the long-run average fuzzy reward when only the cycle is considered since $E[\tilde{X}]$ is the expected length of the cycle.

\section{T-age replacement policy}

The renewal reward theorem is the key tool for obtaining maintenance or replacement strategies which have small long-run cost associated with them. Consider a system of one component which fails over time. The maintenance policy of interest is "Replace the item at time $T$ or at failure, whichever comes first". Such policy might be appropriate, for instance, in the following situation. Consider a production process which gets initiated every day by a switch. The switch fails and needs to be replaced periodically. There are two costs associated with this strategy: $c_{1}$ which is the cost of buying a new switch and an additional cost of $c_{2}$ if the switch fails. Suppose that $c_{1}$ and $c_{2}$ cannot be known precisely. Then it will be more reasonable to assume $c_{1}$ and $c_{2}$ as closed fuzzy numbers $\bar{c}_{1}$ and $\bar{c}_{2}$, respectively.

We say that a cycle is complete every time if a new item is purchased. Let $\tilde{X}$ be the lifetime of the item and be a fuzzy random variable. Then the fuzzy cost incurred during a cycle will be given by 


$$
\tilde{R}=\left\{\begin{array}{cc}
\bar{c}_{1}, & \tilde{X}>T \\
\bar{c}_{1} \oplus \bar{c}_{2}, & \tilde{X} \leq T
\end{array}\right.
$$

Then, we have

$$
\begin{gathered}
\tilde{R}_{\alpha}^{L}=\left\{\begin{array}{cc}
\bar{c}_{1 \alpha}^{L}, & \tilde{X}>T \\
\bar{c}_{1 \alpha}^{L} \oplus \bar{c}_{2 \alpha}^{L}, & \tilde{X} \leq T
\end{array}\right. \\
\tilde{R}_{\alpha}^{U}=\left\{\begin{array}{cc}
\bar{c}_{1 \alpha}^{U}, & \tilde{X}>T \\
\bar{c}_{1 \alpha}^{U} \oplus \bar{c}_{2 \alpha}^{U}, & \tilde{X} \leq T
\end{array}\right.
\end{gathered}
$$

Then

$$
\begin{aligned}
E[\tilde{R}]=\bar{c}_{1} \operatorname{Cr}\{\tilde{X}>T\}+\left(\bar{c}_{1}+\bar{c}_{2}\right) \operatorname{Cr}\{\tilde{X} \leq T\} \\
=\bar{c}_{1}(\operatorname{Cr}\{\tilde{X}>T\}+\operatorname{Cr}\{\tilde{X} \leq T\})+\bar{c}_{2} \operatorname{Cr}\{\tilde{X} \leq T\}, \\
=\bar{c}_{1}+\bar{c}_{2} F_{\tilde{X}}(T)
\end{aligned}
$$

Then

$$
\begin{gathered}
E\left[\tilde{R}_{\alpha}^{L}\right]=\bar{c}_{1 \alpha}^{L}+\bar{c}_{2 \alpha}^{L} F_{\tilde{X} \alpha}^{L}(T), \\
E\left[\tilde{R}_{\alpha}^{U}\right]=\bar{c}_{1 \alpha}^{U}+\bar{c}_{2 \alpha}^{U} F_{\tilde{X} \alpha}^{U}(T),
\end{gathered}
$$

where $F_{\tilde{X}}(t)$ is the cumulative distribution function of the fuzzy random variable of $X$. If $\bar{c}_{1}$ and $\bar{c}_{2}$ are canonical fuzzy numbers then $E\left[\tilde{R}_{\alpha}^{L}\right]$ and $E\left[\tilde{R}_{\alpha}^{U}\right]$ are continuous with respect to $\alpha$. The length of the cycle is

$$
\begin{cases}\tilde{X}, & \tilde{X} \leq T \\ T, & \tilde{X}>T\end{cases}
$$

The expected length of the cycle is

$$
\int_{0}^{T} C r\{\tilde{X} \geq r\} d r+T(1-F(T)),
$$

Therefore by theorem 3.1, we have

$$
\left(\frac{\bar{R}(t)}{t}\right)_{\alpha}^{L}=\frac{\bar{c}_{1 \alpha}^{L}+\bar{c}_{2 \alpha}^{L} F_{\tilde{X} \alpha}^{L}(T)}{E\left[\tilde{X}_{\alpha}^{L}\right]} \text { as } t \rightarrow \infty,
$$

and

$$
\left(\frac{\bar{R}(t)}{t}\right)_{\alpha}^{U}=\frac{\bar{c}_{1 \alpha}^{U}+\bar{c}_{2 \alpha}^{U} F_{\tilde{X} \alpha}^{U}(T)}{E\left[\tilde{X}_{\alpha}^{U}\right]} \text { as } t \rightarrow \infty,
$$

\section{Numerical results}

Suppose that experience shows that the switch will fail approximately between the 0th and 10th day. We then assume that the lifetime of the switch is fuzzy uniform random variable on $(\overline{0}, 1 \overline{0})$. Then

$$
\bar{a}=\overline{0}=(-0.1,0,0.1)
$$

and 


$$
\bar{b}=\overline{10}=(9.5,10,10.5) .
$$

Suppose that $\bar{c}_{1}$ and $\bar{c}_{2}$ are triangular fuzzy numbers with

and

$$
\bar{c}_{1}=\overline{2000}=(1800,2000,2200)
$$

$$
\bar{c}_{2}=\overline{300}=(280,300,320)
$$

Now we have

Then we have

$$
\begin{gathered}
\bar{a}_{\alpha}^{L}=0-0.1 \alpha, \bar{a}_{\alpha}^{U}=0+0.1 \alpha, \bar{b}_{\alpha}^{L}=10-0.5 \alpha, \\
\bar{b}_{\alpha}^{U}=10+0.5 \alpha \text { and } \bar{c}_{1 \alpha}^{L}=2000-200 \alpha, \\
\bar{c}_{1 \alpha}^{U}=2000+200 \alpha, \bar{c}_{2 \alpha}^{L}=300-50 \alpha, \\
\bar{c}_{2 \alpha}^{U}=300+50 \alpha,
\end{gathered}
$$

$$
\begin{aligned}
& E\left[\tilde{X}_{\alpha}^{L}\right]=\frac{10-0.6 \alpha}{2}+T\left(1-F_{\alpha}^{L}(T)\right), \\
& E\left[\tilde{X}_{\alpha}^{U}\right]=\frac{10+0.6 \alpha}{2}+T\left(1-F_{\alpha}^{U}(T)\right) .
\end{aligned}
$$

So,

$$
\begin{aligned}
\left(\frac{\bar{R}(t)}{t}\right)_{\alpha}^{L} & =\frac{\bar{c}_{1 \alpha}^{L}+\bar{c}_{2 \alpha}^{L} F_{\tilde{X} \alpha}^{L}(T)}{\frac{10-0.6 \alpha}{2}+T\left(1-F_{\alpha}^{L}(T)\right)}, \\
\left(\frac{\bar{R}(t)}{t}\right)_{\alpha}^{U} & =\frac{\bar{c}_{1 \alpha}^{U}+\bar{c}_{2 \alpha}^{U} F_{\tilde{X} \alpha}^{U}(T)}{\frac{10+0.6 \alpha}{2}+T\left(1-F_{\alpha}^{U}(T)\right)} .
\end{aligned}
$$

Suppose that $T=7$ years. Then

$$
F_{\tilde{X} \alpha}(7)=\left[\frac{7}{10.6-0.6 \alpha}, \frac{7}{9.9+0.1 \alpha}\right]
$$

Thus,

$$
\begin{aligned}
A_{\alpha}^{L} & =\frac{1800+200 \alpha+(280+20 \alpha)\left(\frac{7}{10.6-0.6 \alpha}\right)}{\frac{9.4+0.6 \alpha}{2}+7\left(1-\frac{7}{10.6-0.6 \alpha}\right)}, \\
A_{\alpha}^{U} & =\frac{2200-200 \alpha+(320-20 \alpha)\left(\frac{7}{9.9+0.1 \alpha}\right)}{\frac{10.6-0.6 \alpha}{2}+7\left(1-\frac{7}{9.9+0.1 \alpha}\right)} .
\end{aligned}
$$

It is easy to see that $A_{\alpha}^{L}$ and $A_{\alpha}^{U}$ are continuous with respect to $\alpha$. Then the membership function of $A$ is 


$$
\mu_{\bar{A}}(r)=\sup _{\alpha \in[0,1]} 1_{\left[A_{\alpha}^{L}, A_{\alpha}^{U}\right]}(r)=\max _{0 \leq \alpha \leq 1} 1_{\left[A_{\alpha}^{L}, A_{\alpha}^{U}\right]}(r)
$$

For the finding the membership function we can use from the following method:

We should solve the nonlinear programming problem [11]

$$
\mu_{\bar{A}}(r)=\max \alpha
$$

Subject to

$$
\begin{aligned}
& \alpha \leq 1, \\
& A_{\alpha}^{L} \leq r, \\
& A_{\alpha}^{U} \geq r, \\
& \alpha \geq 0 .
\end{aligned}
$$

Since $A_{\alpha}^{L}$ is increasing with respect to a and $A_{\alpha}^{U}$ is decreasing with respect to $\alpha$, we have:

(i) if $A_{1}^{L} \leq r \leq A_{1}^{U}$ then $\mu(r)=1$.

(ii) if $r \leq A_{1}^{L}$ then $\mu(r)=\max \left\{\alpha: 0 \leq \alpha \leq 1, a\right.$ is the root of $\left.A_{\alpha}^{L}-r=0\right\}$.

(iii) if $r \geq A_{1}^{U}$ then $\mu(r)=\max \left\{\alpha: 0 \leq \alpha \leq 1, a\right.$ is the root of $\left.A_{\alpha}^{U}-r=0\right\}$.

for this example, the result are

(i) $\quad A_{1}^{L}=A_{1}^{U}=311.268$.

(ii) If $r<311.268$, then the membership of $r$ is $\quad \mu(r)=\frac{r-280.355}{30.911}$.

(iii) If $r>311.268$, then the membership of $r$ is $\quad \mu(r)=\frac{330.105-r}{18.837}$.

Table 1 and figure 1 shows the membership associated with the long-run average cost in this case.

Table 1. Membership function of the long-run average cost for $T=7$

\begin{tabular}{|c|c|}
\hline $\begin{array}{c}\text { Long-run } \\
\text { average cost }\end{array}$ & $\begin{array}{l}\text { Membershi } \\
\mathrm{p} \text { function }\end{array}$ \\
\hline 282 & 0.0532 \\
\hline 285 & 0.1503 \\
\hline 290 & 0.3120 \\
\hline 295 & 0.4737 \\
\hline 300 & 0.6355 \\
\hline
\end{tabular}




\begin{tabular}{|c|c|}
\hline 311.268 & 1.0000 \\
\hline 315 & 0.8019 \\
\hline 320 & 0.5364 \\
\hline 325 & 0.2710 \\
\hline 330 & 0.0056 \\
\hline
\end{tabular}

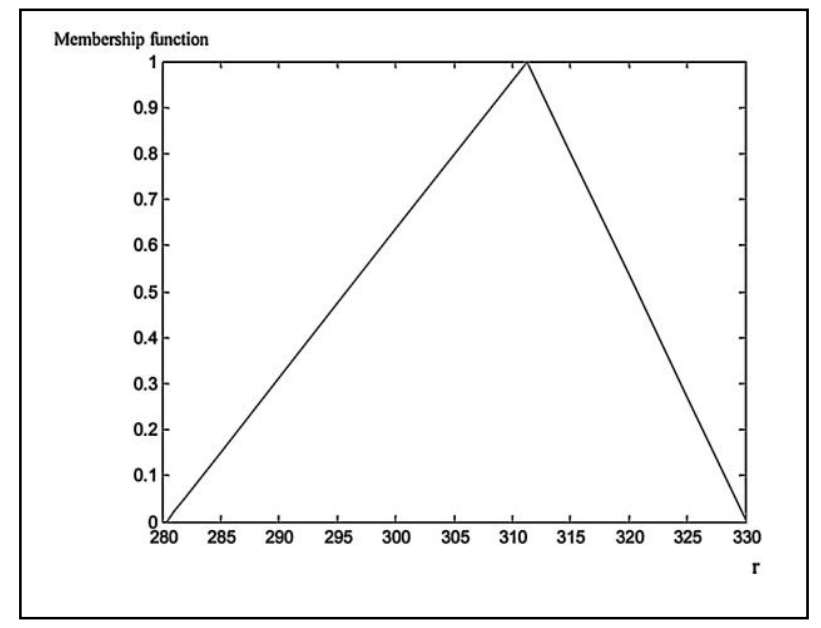

Figure 1. Membership function for the long-run cost when $T=7$

\section{Conclusion}

In this paper, we discussed a renewal reward theorem which shows the asymptotic behavior of the fuzzy lifetime distribution and the fuzzy expected cost per unit time and a numerical procedure to calculate the membership associated with the long-run average fuzzy cost are provided. The presented methodology works for any fuzzy lifetime distribution and closed fuzzy numbers failure costs. In our future work, it could extend this approach to systems with more than one unit and policies like mfailure or block replacement.

\section{References}

[1] R. Zhao, B. Liu, "Renewal process with fuzzy interarrival times and rewards", International Journal of Uncertainty, Fuzziness and Knowledge- Based Systems 11 (2003) 573-586.

[2] S. Wang, J. Watada, "Fuzzy random renewal reward process and its applications", Information Sciences 179 (2009) 4057-4069.

[3] C. Valdez-Florez, R.M. Feldman, "A survey of preventive maintenance models for stochastically deteriorating singleunit systems", Naval Research Logistics 36 (1989) 419-446.

[4] J.J. Buckley, "Fuzzy Probability and Statistics", Studies in Fuzziness and Soft Computing, Volume 196 (2006) 8-12. 
[5] P. Chang, "Fuzzy strategic replacement analysis", European Journal of Operational Research 16(2005) 532-559.

[6] S. Nahmias, "Fuzzy variables", Fuzzy Sets and Systems 1 (1978) 97-110.

[7] B. Liu, "Uncertainty Theory: An Introduction to its Axiomatic Foundation", Springer-Verlag, Berlin, 2004.

[8] B. Liu, Y. Liu, "Expected value of fuzzy variable and fuzzy expected value model", IEEE Transactions on Fuzzy Systems 10 (2002) 445-450.

[9] E. Popova, H.C. Wu, "Renewal reward processes with fuzzy rewards and their applications to Tage replacement policies", European Journal of Operational Research 117 (1999) 606-617.

[10] S.M. Ross, "Stochastic Processes", Wiley, New York, 1983.

[11] M.S. Bazarra, C.M. Shetty, "Nonlinear Programming", Wiley, New York, 1993. 